\title{
A Bayesian approach to reveal the key role of mask wearing in modulating interpersonal distance during the COVID-19 outbreak
}

\author{
Matteo P. Lisi ${ }^{a, b^{*}}$, Marina Scattolin ${ }^{\mathrm{a}, \mathrm{b}}$, Martina Fusaro ${ }^{\mathrm{b}}$, Salvatore Maria Aglioti ${ }^{\mathrm{a}, \mathrm{b}}$ \\ a Sapienza, Università degli Studi di Roma \& CLNS@ Sapienza, Istituto Italiano di
} Tecnologia, 00142, Rome, Italy;

${ }^{\mathrm{b}}$ Social Neuroscience Laboratory, Fondazione Santa Lucia, 00142, Rome, Italy

*To whom correspondence may be addressed:

matteo.lisi@uniroma1.it 


\begin{abstract}
Humans typically create and maintain social bonds through interactions that occur at close social distances. The distance maintained from others is usually less than the one of at least $1.5 \mathrm{~m}$ recommended as a relevant measure for COVID-19 contagion containment. In a webbased experimental study conducted during the first pandemic wave (mid-April 2020), we asked 242 participants to regulate their preferred distance towards confederates who did or did not wear protective masks and gloves and whose COVID-19 test results were positive, negative, or unknown. Information concerning dispositional factors (perceived vulnerability to disease, moral attitudes, and prosocial tendencies) and situational factors (perceived severity of the situation in the country, frequency of physical and virtual social contacts, and attitudes toward quarantine) that may modulate compliance with safety prescriptions was also acquired. A Bayesian analysis approach was adopted. Individual differences did not modulate interpersonal distance. We found strong evidence in favor of a reduction of interpersonal distance towards individuals wearing protective equipment and who tested negative to COVID-19. Importantly, shorter interpersonal distances were maintained towards confederates wearing protective gear, even when their COVID-19 test result was unknown or positive. This protective equipment-related regulation of interpersonal distance may reflect an underestimation of perceived vulnerability to infection; this perception must be discouraged when pursuing individual and collective health-safety measures.
\end{abstract}




\section{A Bayesian approach to reveal the key role of mask wearing in modulating interpersonal distance during the COVID-19 outbreak}

On March 11, 2020, the World Health Organization described the COVID-19 outbreak as a pandemic to signal that the new coronavirus disease had spread across continents, covering large parts of the world. The severe acute respiratory syndrome coronavirus-2 (SARS-CoV2), which is the virus responsible for the emergence of the COVID-19 disease, was found to be transmissible during social interactions, when particles emitted from an infected person's respiratory system may enter another's (Bahl et al., 2020). To limit gatherings and closerange interactions, multiple governments imposed the closure of many public places. These closures and other measures of transmission containment, such as handwashing and use of face masks (World Health Organization, 2020), have been widely adopted in conjunction with maintaining interpersonal distances of at least $1.5 \mathrm{~m}$ (Chu et al., 2020). The need to regulate the minimum distance during in-person interactions is justified by the observation that humans tend to keep themselves about $1 \mathrm{~m}$ from unfamiliar individuals (Hecht, Welsch, Viehoff, \& Longo, 2019), a distance less than the recommended one. Crucially, this distance seems to hold across different countries (Sorokowska et al., 2017), suggesting that the imposed governmental measures sought to change a globally established, everyday behavior. While the reasons behind enforcement of such distancing rules are clear, it remains to be clarified which dispositional and situational factors may impact adherence to interpersonal distancing measures.

Research in proxemics, the study of interpersonal spatial behavior (VandenBos, 2007), has defined interpersonal distance (IPD) as the separation zone that individuals keep between themselves and others (Hayduk, 1978). IPD is shaped by situational factors such as social threat (Vagnoni, Lewis, Tajadura-Jiménez, \& Cardini, 2018) and interpersonal attraction (Welsch et al., 2020), as well as individual characteristics such as morality (Iachini, Pagliaro, 
\& Ruggiero, 2015) and prejudice (Lisi, Fusaro, Tieri, \& Aglioti, 2020). Ultimately, IPD appears to be automatically regulated according to distance-related feelings of personal comfort (Sommer, 2002). Although previous research has helped define IPD under normal circumstances, insights on which factors influence the regulation of IPD during a pandemic are lacking. A limited body of research suggests that greater distances are kept when others are improperly perceived as contagious (i.e., people with AIDS) or as a threat to an individual's health (Mooney, Cohn, \& Swift, 1992; Toppenberg, Bos, Ruiter, Wigboldus, \& Pryor, 2015). Moreover, two studies conducted during the first COVID-19 outbreak (Cartaud, Quesque, \& Coello, 2020; Iachini et al., 2020) showed that a smaller IPD was preferred when the other person was wearing a mask. One possible interpretation is that the sight of a person wearing a face mask triggers a feeling of safety. It is important to note that, if not accompanied by the appropriate IPD, wearing a mask is not in itself sufficient to prevent contagion (World Health Organization, 2020b). Therefore, one crucial aspect to clarify is whether wearing a mask can reduce the IPD even when the other person is contagious. If this is the case, the erroneous belief that use of protective equipment is enough to prevent contagion may have potentially dangerous effects.

It is worth noting that modulations of IPD during a pandemic may not only mirror selfprotective motives, but also affiliative (Dezecache, Frith, \& Deroy, 2020) and cooperative ones (Shim, Chapman, Townsend, \& Galvani, 2012). Understanding which of these variables are more influential on IPD behavior is crucial in the current global context, where policies that effectively reduce contagion are fundamental.

Given the circumstances preventing in-person testing, we used the Interpersonal Visual Analogue Scale (IVAS), a validated, self-report measure of IPD (Iachini et al., 2016). In the IVAS, participants were presented with a silhouette and an avatar's profile on a computer screen. The silhouette represented the participant and the avatar represented a possible 
unknown individual. Participants were asked to indicate the shortest distance between themselves and the other person that they would feel comfortable maintaining. Both male and female interactants were considered, and distance was indicated along a horizontal line. Since the aim of this study was to investigate whether being at risk of infection modulates IPD, the avatar representing the other person was associated with a negative, positive or unknown COVID-19 test result. In addition, the avatar could be wearing protective equipment (i.e., mask and gloves) or not. Factors hypothesized to play a role included (a) the perceived vulnerability to a disease, and (b) the perceived severity of the situation in the country, which were relevant for evaluating attitudes towards the threat itself. We also explored (c) the role of individual differences in levels of physical and virtual contact prior to participation. Finally, we aimed to assess the possible role of different styles of (d) moral thinking (individualization-oriented and binding-oriented), (e) attitudes toward quarantine, and (f) altruism.

We expected participants to maintain a greater distance when others were not wearing protective equipment and when they were identified as positive to COVID-19. The shortest distance was expected to be observed when the other person was wearing a mask and gloves and had received a negative diagnosis of COVID-19. We included a condition in which COVID-19 test results were unknown. This was our control condition and was used to estimate (i) how participants may react to strangers approaching them in everyday situations, and (ii) how reactions may change when the other person is wearing protective equipment or not. We expected higher levels of perceived severity of the situation in the country of participants and their perceived vulnerability to a disease to be associated with greater IPD. As to the affiliative domain, we expected our results to show one of the following two paths: on the one hand, participants who engaged in fewer virtual and physical contact may display a stronger tendency to distance themselves from others (Layden, Cacioppo, \& Cacioppo, 
2018) compared to participants with more frequent contacts. On the other hand, and in accordance with the contact hunger hypothesis (Dezecache et al., 2020), the opposite pattern of results could appear: people who had engaged in more frequent and recent social contacts at the time of testing may not feel the need for closeness that people who had engaged in fewer and less frequent contacts may feel. We expected that participants' positive attitudes toward quarantine may be associated with greater IPD across all conditions. In addition, since binding (vs. individualizing) moral intuitions are more strongly correlated with dispositional germ aversion (Park \& Isherwood, 2011), we expected binding moral thinking styles to contribute to the tendency to maintain a greater IPD. Lastly, if prosocial motives play a role, higher levels of altruism should predict greater distance. In order to avoid overfitting and to select only the relevant variables, we used a model selection approach (Vehtari, Gelman, \& Gabry, 2017).

\section{Materials and Method}

\section{Participants}

A power analysis using MorePower (Campbell \& Thompson, 2012) indicated that a sample size of 238 participants was necessary to detect a small effect size $(\eta 2=0.02)$ with a power of 0.80 . This analysis was performed for a repeated measure design 2 (Participant's Gender: Male/Female) x 2 (Other Avatar's Gender: Male/Female) x 2 (Protective Equipment: Worn/NotWorn) x 3 (COVID-19 Test Result: Positive/Negative/Unknown). Participants were recruited through the online platform Prolific (Palan \& Schitter, 2018) and were compensated with \$2.28 USD (\$6.50 USD per hour) for their participation. Data collection started on April 16, 2020, and ended on April 22, 2020.

Of the original 250 participants, eight were excluded due to failure in two or more attentional checks. A total of 242 international participants (100 women) were included in the final sample. Demographic characteristics of the sample, as well as a list of all countries of 
residence, are presented in Table 1. All countries of residence involved in the data collection had an average Government Stringency Index (a composite measure of the strictness of contagion policies in each country, based on nine response indicators, ranging from 0 to 100 ; Hale et al., 2020) greater than 60 (see Table 1).

\section{Procedure}

The experiment was conducted using PsyToolkit (Stoet, 2010, 2017). After reading general information concerning the study, participants could check the informed consent page and agree to take part in the study. Only those who gave their consent to participate could begin the survey. Participants always completed the demographic information and the questionnaires before the IVAS task. The silhouette representing participants in the IVAS task was selected based on each participant's gender (task version "Female-Self" and "Male-Self"). The silhouette was pictured in a standing position on a marker at the left end of a line and facing the right end of the line (see Figure 1). The height of participants' silhouettes and of the other person's virtual avatar were matched. The virtual avatars were realized using MakeHuman Community 1.2.0 (http://www.makehumancommunity.org); the pictures of the avatars were taken using Unity v.2019.4.15f1 (https://unity.com). Before beginning the IVAS, participants were provided with the following instructions: "Imagine that you are the person on the left of the line and that you cannot turn nor move. Then, imagine that the other person, depicted on the line, begins walking toward you. You should indicate how close you would allow this person to approach you while still being comfortable with that distance. To indicate where the other should stop, click on the horizontal line. Then, press 'Next' to move to the following trial. During the task you will be approached by men and women, that may or may not be wearing masks and gloves. On the top center of the screen you'll read some information about the results of their COVID-19 test: a red sign reporting 'COVID-19 +' indicates a person that tested 
positive; a green 'COVID-19 -' indicates a person whose results were negative; a grey 'COVID-19 ?' indicates that the person was not tested or that results are unknown." In each trial, participants were reminded of these instructions by the sentence: "Click the position on the line where you'd want the other person to stop." This reminder was continuously displayed on the top-center of the screen, above the indicator of COVID-19 Test Result (Figure 1). For each condition (i.e., the combination of factors Other Avatar's Gender x Protective Equipment x COVID-19 Test Result), a total of three trials was presented. Three catch trials were included to ensure that participants were paying attention during the task. In these trials, participants were asked to place the other person's avatar on the far-left end of the line. A total of 39 trials were presented to participants. For each trial, the distance between the participant's silhouette and the other person's avatar was calculated considering that the left end of the horizontal line corresponded to 0 while the right end of the line corresponded to 100.

\section{Measures}

\section{Perceived Severity of the Situation Relative to the COVID-19 Outbreak in the Country}

Perceived Severity of the Situation concerning the COVID-19 outbreak was assessed through participants' answers to the following question: "In your opinion, how serious is the situation related to COVID-19 in your country?" Participants rated this on a VAS ranging from 0 (labelled as not serious at all) to 100 (extremely serious).

\section{Physical and Virtual Contact}

Physical Contact was assessed by means of the question "How often did you have PHYSICAL contacts (for instance, hugs, cuddling, handshakes, etc.) in the last two weeks?" Participants provided their response on a 5-point Likert scale: never (1), rarely (2), sometimes (3), often (4), always (5). Participants rated frequency of Virtual Contact on a single-item, 5point Likert scale: never (1), rarely (2), sometimes (3), often (4), always (5). The question 
was the following: "How often did you have VIRTUAL contacts (for instance, through Skype, Zoom, WhatsApp, etc.) in the last two weeks?"

\section{Moral Foundations Questionnaire (MFQ)}

MFQ is a self-report questionnaire that contains 30 items related to harm, fairness, in-group loyalty, respect for authority, and purity (six items for each foundation; Graham et al., 2011). We computed scores for the individualizing foundations (mean of the harm/care and fairness/reciprocity subscales; Cronbach's $\alpha=0.77$ ) and the binding foundations (mean of the in-group/loyalty, authority/respect, and purity/sanctity subscales; $\alpha=0.85$ ); these values were used in subsequent analyses since we were interested in individualizing and binding foundations broadly. The focus on the well-being of individuals is observed in association with individualizing approaches to moral thinking (Graham, Haidt, \& Nosek, 2009). People who rely on this style of moral thinking focus on protection of individuals from harm and unfairness and consider individuals as the center of moral regulations (Graham et al., 2011). On the other hand, binding foundations favor moral evaluations that are group-oriented and value authority.

\section{Perceived Vulnerability to Disease}

Participants completed an adapted version of a questionnaire assessing individual differences in perceived vulnerability to disease (PVD; Duncan, Schaller, \& Park, 2009). Specifically, we dropped one item ("I avoid using public telephones because of the risk that I may catch something from the previous user") because of its poor relevance to the contemporary context, where the majority of people use cell phones. Consequently, a 14-item version PVD was employed. The overall PVD score (Cronbach's $\alpha=0.75$ ) was used for our analysis.

\section{Public Attitudes Toward Quarantine}

This is a 15-item questionnaire developed by (Tracy, Rea, \& Upshur, 2009). Participants were asked to rate each sentence on a 5-point Likert scale ranging from strongly disagree to 
strongly agree. The Justification subscale (Cronbach's $\alpha=0.68$ ) was entered in the analysis to investigate the relationship between the agreement with the use of quarantine and the interpersonal distance regulation.

\section{Self-Report Altruism Scale}

For assessment of participants' altruism, the scale developed by Rushton and colleagues (1981) was employed. This includes 18 items measuring helping or altruistic traits based on the frequency of helping behaviors. Cronbach's $\alpha$ was 0.84 .

The full survey can be found in the Supplemental Information.

\section{Results}

\section{Model Comparison}

A Bayesian analysis approach was applied. This approach differs from the one used within the standard framework of frequentist null-hypothesis significance testing (NHST) in that it allows evidence to be obtained in favor of the null hypothesis and discrimination between "absence of evidence" and "evidence of absence" (Keysers, Gazzola, \& Wagenmakers, 2020).

Data analyses were computed using the programming language R (R Core Team, 2013) by means of the RStudio interface (RStudio Team, 2015). Preparation and plotting of data were performed using several tidyverse packages (Wickham et al., 2019); modeling and inference were performed using the brms package (Bürkner, 2017), which is based on the probabilistic programming language Stan (Carpenter et al., 2017). Packages emmeans (Lenth, Singmann, Love, Buerkner, \& Herve, 2018) and bayestestR (Makowski, Ben-Shachar, \& Lüdecke, 2019) were employed for computing contrasts between posterior distributions, credible intervals, and Bayes factors. 
The score along the 0-100 Visual Analogue Scale was used as a measure of the distance that participants preferred to keep between themselves and other person's avatars. This measure was set as the outcome of our analysis. We first graphically inspected the univariate and bivariate distributions of outcome and predictor variables. This was done in order to (i) check data distributions and identify potential errors/anomalies, and (ii) identify which models to adopt for a better fit of our data.

To account for the nested structure of our sample, that is, participants nested within countries, multilevel modeling was used (Raudenbush \& Bryk, 2002). Multilevel models of increasing complexity were fitted in order to select the most accurate one. A list of all models can be found in Table 2. The starting point was a "Primary" Model, which included the main effects of COVID-19 Test Result, Protective Equipment, Participant's Gender and Other Avatar's Gender. The interactions between COVID-19 Test Result and Protective Equipment and between Participant's and Other Avatar's Gender were also included as predictors. All models presented here included the intercept over Participants and Countries as random effects (see Table 1).

All continuous predictors were mean-centered. The two ordinal scales Virtual and Physical Contact were dichotomized into "Frequent" (including “Often" and "Always") and "Infrequent” (including "Never," "Rarely," and "Sometimes"). Non-informative, normallydistributed priors $(M=0, S D=1000)$ were applied to all models, on all population-level effects and $t$ - distributed priors $(d f=3, M=0, S D=28)$ on the intercept and on the grouplevel effects. Use of non-informative prior prevents results from being biased toward alternative hypotheses (Weakliem, 1999) and respects the Laplacian principle of indifference (de Laplace, 1825). All models were fitted using four independent Markov chains. Each chain had 30,000 iterations, the first 15,000 of which were warm-up. This led to a total of 60,000 post warm-up posterior samples for inference. According to standard convergence 
diagnostics (Vehtari, Gelman, Simpson, Carpenter, \& Bürkner, 2020), all models converged (Rhat < 1.05) with sufficient precision (effective sample size > 1000).

The models were compared through approximate leave-one-out cross-validation and using Pareto-smoothed importance sampling (PSIS-LOO; Vehtari et al., 2017), which estimates out-of-sample predictive accuracy adopting within-sample fits. Model comparison showed that the model including the same structure of the Primary model plus the main effects of Perceived Severity of the situation in the country, Perceived Vulnerability to Disease, and Physical and Virtual Contact had the best predictive accuracy (see Table 3).

\section{Final Model}

To visualize the probability of direction of the effect for each parameter included in the study, see Figure 2. The summary of the model is reported in Table 4. Table 5 presents contrasts between all levels of Protective Equipment and COVID-19 Test Result; a graphical representation of this interaction can be found in Figure 3. Contrasts between all levels of Participant's Gender and Other Avatar's Gender are reported in Table 6.

Analysis of the final model focused on posterior contrasts between all levels of categorical predictors and the slope of continuous predictors. In order to quantify the uncertainty and magnitude of effects, we computed the $95 \%$ highest density interval (HDI). Any parameter value inside the HDI has higher probability density than any parameter value outside the HDI (Kruschke \& Liddell, 2018). However, the credible interval is conditional on $H 1$ being true and quantifies the strength of an effect, assuming it is present (van Doorn et al., 2020). To quantify evidence for presence or absence of the effects, we computed the Bayes factors (Keysers et al., 2020). The BF quantifies the relative predictive performance of two rival hypotheses, and represents the degree to which data require a change in beliefs concerning the relative plausibility hypotheses (van Doorn et al., 2020). A common rule of thumb is the 
following: $\mathrm{BF}_{10}>3$ indicates support for the alternative hypothesis and $\mathrm{BF}_{10}<0.333$ suggests support for the null hypothesis (van Doorn et al., 2020).

We found strong evidence that the preferred IPD was shorter for the Negative-diagnosed avatar in comparison with the Positive-diagnosed (estimate= -27.90, HDI [-28.65, -27.11], $\mathrm{BF}_{10}=3.526 \mathrm{e}+97$, see Figure 3) and with the Unknown-diagnosed avatar (estimate -11.37 , $95 \%$ HDI $\left.[-12.13,-10.59], \mathrm{BF}_{10}=9.795 \mathrm{e}+42\right)$. The preferred IPD was larger for the Positivediagnosed compared to the Unknown-diagnosed (estimate 16.52, HDI $[15.76,17.30], \mathrm{BF}_{10}=$ 1.458e+47). The preferred IPD was shorter for the Worn Protective Equipment condition compared to the Not Worn Protective Equipment condition (estimate= -6.58, HDI [-7.67, 5.50], $\left.\mathrm{BF}_{10}=1.08 \mathrm{E}+11\right)$. This effect was present when considering the Negative-diagnosed avatar, (estimate $=-6.58$, HDI $\left.[-7.67,-5.50], \mathrm{BF}_{10}=2.68 \mathrm{E}+09\right)$, the Unknown-diagnosed avatar $\left(\right.$ estimate $\left.=-6.41, \mathrm{HDI}[-7.48,-5.30], \mathrm{BF}_{10}=8.81 \mathrm{E}+08\right)$ and Positive-diagnosed avatar (estimate $=-7.60$, HDI $\left.[-8.69,-6.52], \mathrm{BF}_{10}=1.08 \mathrm{E}+13\right)$.

We also found non-zero effects for Participant's Gender and Virtual Contact. However, in both cases the Bayes factor indicated moderate evidence in support of the null hypothesis: the preferred IPD was shorter for women compared to men (estimate $=5.08$, HDI $[.08,10.06]$, $\left.\mathrm{BF}_{10}=0.02\right)$ and for participants who had Infrequent Virtual Contact during the two weeks prior to participation compared to participants who had Frequent Virtual Contact (estimate= 8.71, HDI [-14.23, -3.07], $\left.\mathrm{BF}_{10}=.28\right)$. No other credible effects were found.

\section{Discussion}

Interpersonal distance of at least $1.5 \mathrm{~m}$ is a fundamental measure of containment for the spreading of SARS-CoV-2. Adherence to this measure represents a dramatic change from people's behavior under normal circumstances. As of now, the dispositional and situational factors that impact adherence to this rule are under-investigated. In this study, we explored the role of protective equipment, actual risk of infection, perceived vulnerability, severity of 
the situation, physical and virtual contacts, morality, attitudes toward quarantine, and prosocial tendencies in the regulation of IPD during the COVID-19 outbreak. Using a model selection approach, we aimed to identify the most relevant variables to predict IPD behavior. In line with previous studies that investigated the distance maintained from infected individuals (Mooney et al., 1992; Toppenberg et al., 2015), we found strong evidence that providing information regarding a positive COVID-19 test result increased IPD. In particular, we found a continuous increase in the space that participants put between themselves and another person, ranging from the shortest distance from a negative-tested individual to a maximum distance when the other tested positive to COVID-19. These results may reflect the notion of a "behavioral immune system" (Ackerman, Hill, \& Murray, 2018), according to which humans use behavioral avoidance of disease-causing objects and people as a diseasemanagement strategy in addition to their own immune systems.

We also found strong evidence that interacting with someone who was wearing protective equipment was associated with reduced IPD. This result is consistent with the findings of other studies conducted during the COVID-19 pandemic that used different methodologies (Cartaud et al., 2020; Iachini et al., 2020). Specifically, Iachini and colleagues (Iachini et al., 2020) used an 8-point Likert scale (ranging from $1=0.5 \mathrm{~m}$ to $8=4 \mathrm{~m}$ ) and found that the comfort-distance from others wearing a mask was shorter than the one from others without a mask. Cartaud and colleagues (Cartaud et al., 2020) used characters showing either positive, neutral, or negative facial expression or wearing a mask (which was always associated to a neutral facial expression). Characters were presented at different fixed distances from participants. Results showed that shorter IPD was judged as more appropriate for the character wearing a mask compared to the other conditions. It has been suggested that the sight of a mask could induce a feeling of safety that facilitates a reduction of IPD. Enactment of this tendency in real-life situations may constitute a potential threat, since the use of 
protective equipment alone is not enough to prevent SARS-CoV-2 from spreading (World Health Organization, 2020b). Therefore, one question that remained unanswered is whether the reduction of IPD in response to the sight of protective equipment also occurs when there is an actual risk of infection. Importantly, at variance with the previous studies, we observed that the effect of mask wearing is present not only when participants are not provided with information regarding the other person's COVID-19 test result, but also when they are provided with information of a positive diagnosis. In public settings, allowing a shorter IPD to a person wearing protective equipment may create conditions for further transmission of the new coronavirus.

Among the other predictors, only Virtual Contact had a non-zero effect: frequent virtual contact led to larger IPD compared to infrequent virtual contact. However, it is worth noting that the Bayes factor analysis did not reveal support for this effect. According to the contact hunger hypothesis, the type of social isolation individuals may experience during the pandemic could lead to an enhanced need for physical contact (Dezecache et al., 2020). Indeed, affiliation and contact-seeking are core responses to perceived danger (Dezecache, Grèzes, \& Dahl, 2017; Mawson, 2017), and this may happen even in cases where contact itself is a threat, as in infectious diseases. It is possible that engaging in frequent virtual contacts may have modulated this evolutionary drive, leading to lower motivation for interpersonal connection in comparison to those who experienced infrequent virtual contact. Future studies should systematically investigate the effect of virtual contact's quantity and quality in modulating IPD during social isolation.

The present findings are limited by the projective nature of the task and must be replicated in a more ecological context. Moreover, future studies are needed to clarify whether the reduction of IPD following the sight of worn protective equipment is present across different public context (i.e., hospitals, supermarkets) and whether this effect is dependent on the 
social encoding of the other person. Finally, the results of our study must be considered in light of recent neuroscientific evidence (Vieira, Pierzchajlo, \& Mitchell, 2020), which shows that the distance from other organisms (conspecific or not) is regulated by a common network that includes the midbrain periaqueductal gray (a defensive region sensitive to threat proximity) and frontoparietal structures representing peripersonal space (the multisensory motor area within which it is possible to reach and interact with objects; (di Pellegrino \& Làdavas, 2015). Neuroimaging studies could investigate whether the reduction of IPD given by the sight of wearing a mask reflects a modulation of the activity in this network, therefore supporting the hypothesis of a reduced perceived threat.

\section{Declarations}

\section{Funding}

This work was supported by a European Research Council (ERC) Advanced Grant 2017, Embodied Honesty in real world and digital interactions (eHONESTY) (to S.M. Aglioti) and Avvio alla Ricerca (2019) awarded by La Sapienza University of Rome to M. Fusaro.

\section{Conflict of interest}

The authors declare that they have no financial or other conflicts of interest.

\section{Ethics approval}

All procedures were approved by the Ethics Committee of the Department of Psychology, University of Rome "La Sapienza" (Prot. n. 0000612) and in accordance with the ethical standards laid down in the Declaration of Helsinki (2013).

\section{Consent to participate}

Informed consent was obtained from all participants.

\section{Availability of data}

The raw data and scripts for the analysis will be available on a public data repository at the moment of publication of the manuscript in a peer-reviewed journal. 


\section{Author Contributions}

M.P.L., M.S., M.F., and S.M.A. conceived and designed research; M.P.L. prepared the virtual avatars; M.S. prepared the Psytoolkit experiment; M.P.L., M.S., M.F. performed experiments; M.P.L. analyzed data; M.P.L. drafted manuscript; M.P.L., M.S., M.F. and S.M.A. edited and revised and approved final version of manuscript.

\section{Open practice statement}

The work reported here was not preregistered.

\section{References}

Ackerman, J. M., Hill, S. E., \& Murray, D. R. (2018). The behavioral immune system: Current concerns and future directions. Social and Personality Psychology Compass, 12(2), e12371. https://doi.org/10.1111/spc3.12371

Bahl, P., Doolan, C., de Silva, C., Chughtai, A. A., Bourouiba, L., \& MacIntyre, C. R. (2020). Airborne or Droplet Precautions for Health Workers Treating Coronavirus Disease 2019? The Journal of Infectious Diseases, jiaa189. https://doi.org/10.1093/infdis/jiaa189

Bürkner, P.-C. (2017). brms: An $R$ Package for Bayesian Multilevel Models Using Stan. Journal of Statistical Software, 80(1). https://doi.org/10.18637/jss.v080.i01

Campbell, J. I. D., \& Thompson, V. A. (2012). MorePower 6.0 for ANOVA with relational confidence intervals and Bayesian analysis. Behavior Research Methods, 44(4), 1255-1265. https://doi.org/10.3758/s13428-012-0186-0

Carpenter, B., Gelman, A., Hoffman, M. D., Lee, D., Goodrich, B., Betancourt, M., ... Riddell, A. (2017). Stan: A Probabilistic Programming Language. Journal of Statistical Software, 76(1). https://doi.org/10.18637/jss.v076.i01 
Cartaud, A., Quesque, F., \& Coello, Y. (2020). Wearing a face mask against Covid-19 results in a reduction of social distancing. PLOS ONE, 15(12), e0243023. https://doi.org/10.1371/journal.pone.0243023

Chu, D. K., Akl, E. A., Duda, S., Solo, K., Yaacoub, S., Schünemann, H. J., ... Schünemann, H. J. (2020). Physical distancing, face masks, and eye protection to prevent person-toperson transmission of SARS-CoV-2 and COVID-19: A systematic review and metaanalysis. The Lancet, 395(10242), 1973-1987. https://doi.org/10.1016/S01406736(20)31142-9

de Laplace, P. S. (1825). Essai philosophique sur les probabilités. Bachelier.

Dezecache, G., Frith, C. D., \& Deroy, O. (2020). Pandemics and the great evolutionary mismatch. Current Biology, 30(10), R417-R419. https://doi.org/10.1016/j.cub.2020.04.010

Dezecache, G., Grèzes, J., \& Dahl, C. D. (2017). The nature and distribution of affiliative behaviour during exposure to mild threat. Royal Society Open Science, 4(8), 170265. https://doi.org/10.1098/rsos.170265

di Pellegrino, G., \& Làdavas, E. (2015). Peripersonal space in the brain. Neuropsychologia, 66, 126-133. https://doi.org/10.1016/j.neuropsychologia.2014.11.011

Duncan, L. A., Schaller, M., \& Park, J. H. (2009). Perceived vulnerability to disease: Development and validation of a 15-item self-report instrument. Personality and Individual Differences, 47(6), 541-546. https://doi.org/10.1016/j.paid.2009.05.001

Graham, J., Haidt, J., \& Nosek, B. A. (2009). Liberals and conservatives rely on different sets of moral foundations. Journal of Personality and Social Psychology, 96(5), 10291046. https://doi.org/10.1037/a0015141 
Graham, J., Nosek, B. A., Haidt, J., Iyer, R., Koleva, S., \& Ditto, P. H. (2011). Mapping the moral domain. Journal of Personality and Social Psychology, 101(2), 366-385. https://doi.org/10.1037/a0021847

Hale, T., Angrist, N., Kira, B., Petherick, A., Philips, T., \& Webster, S. (2020, maggio 25). Variation in Government Responses to COVID-19 Version 6.0. Blavatnik School of Government Working Paper. Retrieved from: www.bsg.ox.ac.uk/covidtracker

Hayduk, L. A. (1978). Personal space: An evaluative and orienting overview. Psychological Bulletin, 85(1), 117-134. https://doi.org/10.1037/0033-2909.85.1.117

Hecht, H., Welsch, R., Viehoff, J., \& Longo, M. R. (2019). The shape of personal space. Acta Psychologica, 193, 113-122. https://doi.org/10.1016/j.actpsy.2018.12.009

Iachini, T., Coello, Y., Frassinetti, F., Senese, V. P., Galante, F., \& Ruggiero, G. (2016). Peripersonal and interpersonal space in virtual and real environments: Effects of gender and age. Journal of Environmental Psychology, 45, 154-164. https://doi.org/10.1016/j.jenvp.2016.01.004

Iachini, T., Frassinetti, F., Ruotolo, F., Sbordone, F. L., Ferrara, A., Arioli, M., ... Ruggiero, G. (2020). Psychological and situational effects on social distancing and well-being during the COVID-19 pandemic: Not a question of real risk [Preprint]. PsyArXiv. https://doi.org/10.31234/osf.io/r7usv

Iachini, T., Pagliaro, S., \& Ruggiero, G. (2015). Near or far? It depends on my impression: Moral information and spatial behavior in virtual interactions. Acta Psychologica, 161, 131-136. https://doi.org/10.1016/j.actpsy.2015.09.003

Keysers, C., Gazzola, V., \& Wagenmakers, E.-J. (2020). Using Bayes factor hypothesis testing in neuroscience to establish evidence of absence. Nature Neuroscience, 23(7), 788-799. https://doi.org/10.1038/s41593-020-0660-4 
Kruschke, J. K., \& Liddell, T. M. (2018). The Bayesian New Statistics: Hypothesis testing, estimation, meta-analysis, and power analysis from a Bayesian perspective. Psychonomic Bulletin \& Review, 25(1), 178-206. https://doi.org/10.3758/s13423-016$1221-4$

Layden, E. A., Cacioppo, J. T., \& Cacioppo, S. (2018). Loneliness predicts a preference for larger interpersonal distance within intimate space. PLOS ONE, 13(9), e0203491. https://doi.org/10.1371/journal.pone.0203491

Lenth, R., Singmann, H., Love, J., Buerkner, P., \& Herve, M. (2018). Emmeans: Estimated marginal means, aka least-squares means. R package version(1(1)), 3.

Lisi, M., Fusaro, M., Tieri, G., \& Aglioti, S. M. (2020). Humans adjust virtual comfortdistance towards an artificial agent depending on their sexual orientation and implicit prejudice against gay men. Completed, unpublished study.

Makowski, D., Ben-Shachar, M., \& Lüdecke, D. (2019). bayestestR: Describing Effects and their Uncertainty, Existence and Significance within the Bayesian Framework. Journal of Open Source Software, 4(40), 1541. https://doi.org/10.21105/joss.01541

Mawson, A. R. (2017). Mass panic and social attachment: The dynamics of human behavior. Routledge.

Mooney, K. M., Cohn, E. S., \& Swift, M. B. (1992). Physical Distance and AIDS: Too Close for Comfort?1. Journal of Applied Social Psychology, 22(18), 1442-1452. https://doi.org/10.1111/j.1559-1816.1992.tb00959.x

Park, J. H., \& Isherwood, E. (2011). Effects of Concerns About Pathogens on Conservatism and Anti-Fat Prejudice: Are They Mediated by Moral Intuitions? The Journal of Social Psychology, 151(4), 391-394. https://doi.org/10.1080/00224545.2010.481692

R Core Team. (2013). R: A language and environment for statistical computing. 
Raudenbush, S. W., \& Bryk, A. S. (2002). Hierarchical linear models: Applications and data analysis methods (Vol. 1). Sage.

RStudio Team. (2015). RStudio: Integrated development for R.

Rushton, J. P., Chrisjohn, R. D., \& Cynthia Fekken, G. (1981). The altruistic personality and the self-report altruism scale. Personality and Individual Differences, 2(4), 293-302. https://doi.org/10.1016/0191-8869(81)90084-2

Shim, E., Chapman, G. B., Townsend, J. P., \& Galvani, A. P. (2012). The influence of altruism on influenza vaccination decisions. Journal of The Royal Society Interface, 9(74), 2234-2243. https://doi.org/10.1098/rsif.2012.0115

Sommer, R. (2002). Personal space in a digital age. In Handbook of environmental psychology. John Wiley \& Sons, Inc..

Sorokowska, A., Sorokowski, P., Hilpert, P., Cantarero, K., Frackowiak, T., Ahmadi, K., ... Pierce, J. D. (2017). Preferred Interpersonal Distances: A Global Comparison. Journal of Cross-Cultural Psychology, 48(4), 577-592. https://doi.org/10.1177/0022022117698039

Stoet, G. (2010). PsyToolkit: A software package for programming psychological experiments using Linux. Behavior Research Methods, 42(4), 1096-1104. https://doi.org/10.3758/BRM.42.4.1096

Stoet, G. (2017). PsyToolkit: A Novel Web-Based Method for Running Online Questionnaires and Reaction-Time Experiments. Teaching of Psychology, 44(1), 2431. https://doi.org/10.1177/0098628316677643

Toppenberg, H. L., Bos, A. E. R., Ruiter, R. A. C., Wigboldus, D. H. J., \& Pryor, J. B. (2015). HIV-related stigma in social interactions: Approach and avoidance behaviour in a virtual environment: HIV-related stigma in social interactions. European Journal of Social Psychology, 45(2), 169-179. https://doi.org/10.1002/ejsp.2082 
Tracy, C. S., Rea, E., \& Upshur, R. E. (2009). Public perceptions of quarantine: Communitybased telephone survey following an infectious disease outbreak. BMC Public Health, 9(1), 470. https://doi.org/10.1186/1471-2458-9-470

Vagnoni, E., Lewis, J., Tajadura-Jiménez, A., \& Cardini, F. (2018). Listening to a conversation with aggressive content expands the interpersonal space. PLOS ONE, 13(3), e0192753. https://doi.org/10.1371/journal.pone.0192753

van Doorn, J., van den Bergh, D., Böhm, U., Dablander, F., Derks, K., Draws, T., ... Wagenmakers, E.-J. (2020). The JASP guidelines for conducting and reporting a Bayesian analysis. Psychonomic Bulletin \& Review. https://doi.org/10.3758/s13423020-01798-5

VandenBos, G. R. (2007). APA dictionary of psychology.

Vehtari, A., Gelman, A., \& Gabry, J. (2017). Practical Bayesian model evaluation using leave-one-out cross-validation and WAIC. Statistics and Computing, 27(5), 14131432. https://doi.org/10.1007/s11222-016-9696-4

Vehtari, A., Gelman, A., Simpson, D., Carpenter, B., \& Bürkner, P.-C. (2020). RankNormalization, Folding, and Localization: An Improved \$lwidehat $\{\mathrm{R}\}$ \$ for Assessing Convergence of MCMC. Bayesian Analysis. https://doi.org/10.1214/20-BA1221

Vieira, J. B., Pierzchajlo, S. R., \& Mitchell, D. G. V. (2020). Neural correlates of social and non-social personal space intrusions: Role of defensive and peripersonal space systems in interpersonal distance regulation. Social Neuroscience, 15(1), 36-51. https://doi.org/10.1080/17470919.2019.1626763

Weakliem, D. L. (1999). A Critique of the Bayesian Information Criterion for Model Selection. Sociological Methods \& Research, 27(3), 359-397. https://doi.org/10.1177/0049124199027003002 
Welsch, R., von Castell, C., Rettenberger, M., Turner, D., Hecht, H., \& Fromberger, P. (2020). Sexual attraction modulates interpersonal distance and approach-avoidance movements towards virtual agents in males. PLOS ONE, 15(4), e0231539. https://doi.org/10.1371/journal.pone.0231539

Wickham, H., Averick, M., Bryan, J., Chang, W., McGowan, L., François, R., ... Yutani, H. (2019). Welcome to the Tidyverse. Journal of Open Source Software, 4(43), 1686. https://doi.org/10.21105/joss.01686

World Health Organization. (2020a). Https://www.who.int/fr/emergencies/ diseases/novelcoronavirus-2019/advice-for-public.

World Health Organization. (2020b). Rational use of personal protective equipment for coronavirus disease 2019 ( COVID-19).

\section{Table 1}

Demographic characteristics for each of the 28 countries of residence included in the study

\begin{tabular}{|c|c|c|c|c|c|c|c|}
\hline \multirow[t]{3}{*}{ Country of residence } & \multicolumn{3}{|c|}{ Gender } & \multicolumn{3}{|c|}{ Age Range } & $\begin{array}{c}\text { Government Stringency } \\
\text { Index }\end{array}$ \\
\hline & Total & Male & Female & $18-34$ & $35-55$ & 55 & Mean (between 16-22 \\
\hline & & & & & & or more & April 2020) \\
\hline Australia & 4 & 4 & 0 & 3 & 1 & 0 & 70.5 \\
\hline Austria & 3 & 1 & 2 & 3 & 0 & 0 & 77.8 \\
\hline Belgium & 2 & 1 & 1 & 2 & 0 & 0 & 81.5 \\
\hline Canada & 3 & 3 & 0 & 3 & 0 & 0 & 72.7 \\
\hline
\end{tabular}




\section{Country of residence}

Gender

Age Range

Government Stringency

Index

\begin{tabular}{cccccc}
\hline Total Male & Female & $18-34$ & $35-55$ & 55 & Mean (between 16-22 \\
& & & or more & April 2020)
\end{tabular}

\begin{tabular}{|c|c|c|c|c|c|c|c|}
\hline Czech Republic & 4 & 4 & 0 & 4 & 0 & 0 & 67 \\
\hline Denmark & 1 & 0 & 1 & 1 & 0 & 0 & 68.5 \\
\hline Estonia & 8 & 2 & 6 & 7 & 1 & 0 & 77.8 \\
\hline Finland & 2 & 1 & 1 & 2 & 0 & 0 & 61.8 \\
\hline France & 7 & 5 & 2 & 6 & 1 & 0 & 87.9 \\
\hline Germany & 4 & 3 & 1 & 4 & 0 & 0 & 76.8 \\
\hline Greece & 16 & 12 & 4 & 13 & 3 & 0 & 84.2 \\
\hline Hungary & 9 & 5 & 4 & 8 & 1 & 0 & 76.8 \\
\hline Ireland & 3 & 2 & 1 & 3 & 0 & 0 & 90.7 \\
\hline Israel & 3 & 3 & 0 & 3 & 0 & 0 & 89.9 \\
\hline Italy & 26 & 15 & 11 & 24 & 2 & 0 & 93.5 \\
\hline Latvia & 2 & 0 & 2 & 2 & 0 & 0 & 69.4 \\
\hline Mexico & 2 & 2 & 0 & 2 & 0 & 0 & 82.4 \\
\hline Netherlands & 4 & 3 & 1 & 4 & 0 & 0 & 79.6 \\
\hline New Zealand & 3 & 2 & 1 & 3 & 0 & 0 & 96.3 \\
\hline
\end{tabular}




\begin{tabular}{|c|c|c|c|c|c|c|c|}
\hline \multirow[t]{3}{*}{ Country of residence } & \multicolumn{3}{|c|}{ Gender } & \multicolumn{3}{|c|}{ Age Range } & \multirow{2}{*}{$\begin{array}{l}\text { Government Stringency } \\
\text { Index } \\
\text { Mean (between } 16-22\end{array}$} \\
\hline & Total & Male & Female & $18-34$ & $35-55$ & 55 & \\
\hline & & & & & & or more & April 2020) \\
\hline Norway & 2 & 2 & 0 & 2 & 0 & 0 & 76.4 \\
\hline Poland & 34 & 23 & 11 & 31 & 3 & 0 & 83.3 \\
\hline Portugal & 30 & 17 & 13 & 22 & 6 & 2 & 82.4 \\
\hline Slovenia & 5 & 4 & 1 & 4 & 1 & 0 & 88.2 \\
\hline South Africa & 1 & 0 & 1 & 0 & 1 & 0 & 87.9 \\
\hline Spain & 10 & 7 & 3 & 8 & 2 & 0 & 85.1 \\
\hline Sweden & 1 & 1 & 0 & 1 & 0 & 0 & 64.8 \\
\hline UK & 48 & 15 & 33 & 31 & 13 & 4 & 79.6 \\
\hline USA & 5 & 5 & 0 & 4 & 1 & 0 & 72.7 \\
\hline Total & 242 & 142 & 100 & 200 & 36 & 6 & \\
\hline
\end{tabular}

Table 2

Formulas for each model

Model Formula

Model $0 \quad$ Distance $\sim 1+(1 \mid$ Country $)+(1 \mid$ Participant $)$ 
Model 1

Distance $\sim 1+(1 \mid$ Country $)+(1 \mid$ Participant $)+$ Protective Equipment $\times$ COVID-19 Test

Result + Participant's Gender $\times$ Other Avatar's Gender $+(1 \mid$ Country $)+(1$

|Participant)

Model 2 Distance $\sim 1+(1 \mid$ Country $)+(1 \mid$ Participant $)+$ Protective Equipment $\times$ COVID-19 Test

Result + Participant's Gender $\times$ Other Avatar's Gender + Perceived Severity of the situation in the Country $+(1 \mid$ Country $)+(1 \mid$ Participant $)$

Model 3 Distance $\sim 1+(1 \mid$ Country $)+(1 \mid$ Participant $)+$ Protective Equipment $\times$ COVID-19 Test Result + Participant's Gender $\times$ Other Avatar's Gender + Perceived Severity of the situation in the Country + Physical Contact + Virtual Contact $+(1 \mid$ Country $)+(1$ |Participant)

Model 4 Distance $\sim 1+(1 \mid$ Country $)+(1 \mid$ Participant $)+$ Protective Equipment $\times$ COVID-19 Test Result + Participant's Gender $\times$ Other Avatar's Gender + Perceived Severity of the situation in the Country + Physical Contact + Virtual Contact + Perceived Vulnerability to Disease $+(1 \mid$ Country $)+(1 \mid$ Participant $)$

Model 5 Distance $\sim 1+(1 \mid$ Country $)+(1 \mid$ Participant $)+$ Protective Equipment $\times$ COVID-19 Test Result + Participant's Gender $\times$ Other Avatar's Gender + Perceived Severity of the situation in the Country + Physical Contact + Virtual Contact + Perceived Vulnerability to Disease + Individualizing Moral Foundation + Binding Moral Foundation + (1) Country) $+(1 \mid$ Participant $)$

Model 6 Distance $\sim 1+(1 \mid$ Country $)+(1 \mid$ Participant $)+$ Protective Equipment $\times$ COVID-19 Test Result + Participant's Gender $\times$ Other Avatar's Gender + Perceived Severity of the situation in the Country + Physical Contact + Virtual Contact + Perceived Vulnerability 
to Disease + Individualizing Moral Foundation + Binding Moral Foundation + Quarantine's Justification $+(1 \mid$ Country $)+(1 \mid$ Participant $)$

Model 7 Distance $\sim 1+(1 \mid$ Country $)+(1 \mid$ Participant $)+$ Protective Equipment $\times$ COVID-19 Test Result + Participant's Gender $\times$ Other Avatar's Gender + Perceived Severity of the situation in the Country + Physical Contact + Virtual Contact + Perceived Vulnerability to Disease + Individualizing Moral Foundation + Binding Moral Foundation + Quarantine's Justification + Altruism + (1| Country $)+(1 \mid$ Participant $)$

\section{Table 3}

Model comparison via Leave-One-Out Cross-Validation

\begin{tabular}{cccc}
\hline Model & ELPD-diff & SE-diff & weight \\
\hline Model 4 & 0 & 0 & 0.19 \\
Model 2 & -0.3 & 0.4 & 0.14 \\
Model 3 & -0.3 & 0.1 & 0.14 \\
Model 1 & -0.3 & 0.5 & 0.13 \\
Model 6 & -0.3 & 0.4 & 0.13 \\
Model 5 & -0.4 & 0.3 & 0.12 \\
Model 7 & -0.5 & 0.4 & 0.11 \\
Model 0 & -2200.3 & 58.1 & 0 \\
\hline
\end{tabular}


Note. We report the differences in the point estimates (ELPD-diff) and standard errors of the difference (SE-diff) of the expected log pointwise predictive density (ELPD). The values in the elpd_diff and se_diff columns of the returned matrix are computed by making pairwise comparisons between each model and the model with the largest ELPD (the model in the first row). ELPD indicates the predictive performance of the model. Model weights are calculated via stacking of the predictive distributions: The method combines all models by maximizing the leave-one-out predictive density of the combination distribution.

\section{Table 4}

Summary of the final model

\begin{tabular}{ccccccc}
\hline Parameter & Median & 95\% HDI & BF 10 & ESS & $\hat{\mathrm{R}}$ \\
& & & & & & \\
& & & & & & \\
\hline Intercept & 33.61 & {$[28.11,39.25]$} & $8.33 \mathrm{E}+10$ & 9319 & 1
\end{tabular}

Protective Equipment

$\begin{array}{cccccc}\text { Worn v. Not Worn } & -6.58 & {[-7.67,-5.50]} & \mathbf{1 . 0 8 E + 1 1} & 33152 & 1 \\ \text { COVID-19 test result } & & & & & \\ \text { Negative v. Positive } & -27.90 & {[-28.66,-27.11]} & \mathbf{3 . 5 2 6 e + 9 7} & 35176 & 1 \\ \text { Negative v. Unknown } & -11.37 & -[12.14,-10.59] & \mathbf{9 . 7 9 5 e + 4 2} & 35998 & 1 \\ & & & & & \\ \text { Positive v. Unknown } & 16.52 & {[15.76,17.30]} & \mathbf{1 . 4 5 8 e + 4 7} & 35765 & 1\end{array}$

Participant's Gender

Female v. Male

5.08

$[0.08,10.06]$

0.02

$6970 \quad 1$

Other Avatar's Gender

0.40

$[-0.39,1.22]$

6.759e-04 $39129 \quad 1$ 


\begin{tabular}{|c|c|c|c|c|c|}
\hline Parameter & Median & $95 \% \mathrm{HDI}$ & $\mathrm{BF}_{10}$ & ESS & $\hat{\mathrm{R}}$ \\
\hline \multicolumn{6}{|l|}{ Male v. Female } \\
\hline \multicolumn{6}{|l|}{ Perceived Severity of the } \\
\hline situation in the Country & 0.10 & {$[-0.01,0.22]$} & $2.773 \mathrm{e}-04$ & 9688 & 1 \\
\hline \multicolumn{6}{|l|}{ Virtual contact } \\
\hline Infrequent v. Frequent & -8.71 & {$[-14.23,-3.07]$} & 0.28 & 10351 & 1 \\
\hline \multicolumn{6}{|l|}{ Physical contact } \\
\hline Infrequent v. Frequent & 5.04 & {$[-0.38,10.28]$} & 0.015 & 9056 & 1 \\
\hline \multicolumn{6}{|l|}{ Perceived Vulnerability to } \\
\hline Disease & -1.04 & {$[-5.99,3.85]$} & 0.003 & 8344 & 1 \\
\hline
\end{tabular}

Note. For all categorical predictors, we report the contrasts between each level of the factor; for the continuous predictors, we report the regression coefficient which represents the change in the outcome resulting from a unit change in the predictor. Results are described by means of the Median, the 95\% HDI and the BF. A BF greater than 3 (highlighted in bold) indicates a strong support for the alternative hypothesis, while the HDI quantifies the magnitude of the effect and its uncertainty.

\section{Table 5}

Contrasts between all levels of Protective Equipment and COVID-19 Test Result 


\begin{tabular}{|c|c|c|c|}
\hline Protective Equipment: COVID-19 test & Median & $95 \% \mathrm{HDI}$ & $\mathrm{BF}_{10}$ \\
\hline \multicolumn{4}{|l|}{ result Contrasts } \\
\hline Negative: Worn v. Not Worn & -6.58 & {$[-7.67,-5.50]$} & $2.68 \mathrm{E}+09$ \\
\hline Unknown: Worn v. Not Worn & -6.41 & {$[-7.48,-5.30]$} & $8.81 E+08$ \\
\hline Positive: Worn v. Not Worn & -7.60 & {$[-8.69,-6.52]$} & $1.08 E+13$ \\
\hline Worn: Negative v. Unknown & -11.46 & {$[-12.55,-10.38]$} & $6.6 \mathrm{E}+19$ \\
\hline Worn: Positive v. Unknown & 15.94 & {$[14.84,17.01]$} & $1.72 E+79$ \\
\hline Worn: Negative v. Positive & -27.39 & {$[-28.48,-26.30]$} & $1.05 \mathrm{E}+70$ \\
\hline Not Worn: Negative v. Unknown & -4.88 & {$[-5.96,-3.78]$} & 645871.4 \\
\hline Not Worn: Positive v. Unknown & 17.13 & {$[16.03,18.21]$} & 1.19E+31 \\
\hline Not Worn: Negative v. Positive & -28.42 & {$[-29.49,-27.31]$} & $2.14 E+78$ \\
\hline $\begin{array}{l}\text { Worn x Negative v. } \\
\text { Not Worn x Positive }\end{array}$ & -35.00 & {$[-36.09,-33.90]$} & $1.34 \mathrm{E}+86$ \\
\hline Not Worn x Negative v. & -20.81 & {$[-21.91,-19.75]$} & $7.18 \mathrm{E}+53$ \\
\hline & -17.87 & {$[-18.94,-16.76]$} & $1.27 \mathrm{E}+44$ \\
\hline Not Worn x Negative v. & -11.28 & {$[-12.39,-10.22]$} & $7.35 \mathrm{E}+24$ \\
\hline
\end{tabular}




\begin{tabular}{cccc}
\hline $\begin{array}{l}\text { Protective Equipment: COVID-19 test } \\
\text { result Contrasts }\end{array}$ & Median & BF $_{10}$ \\
\hline Worn x Positive v. & 9.52 & {$[8.44,10.60]$} & $\mathbf{5 . 8 7 E + 2 0}$ \\
Not Worn x Unknown & & & \\
Not Worn x Positive v. & 23.53 & {$[22.43,24.61]$} & $\mathbf{1 . 4 E + 6 3}$ \\
Worn x Unknown & & & \\
\hline
\end{tabular}

Note. Results are described by means of the Median, the $95 \% \mathrm{HDI}$ and the $\mathrm{BF}_{10}\left(\mathrm{BF}_{10}>3\right.$ highlighted in bold).

\section{Table 6}

Contrasts between all levels of Participant's and Other Avatar's Gender

Participant's Gender: Other Avatar's Gender $\quad$ Median
Contrasts

\begin{tabular}{|c|c|c|c|}
\hline Female Participants: Female Avatar v. Male Avatar & 0.36 & {$[-1.35,0.61]$} & $4.557 \mathrm{e}-04$ \\
\hline Male Participants: Female Avatar v. Male Avatar & -0.41 & {$[-1.23,0.39]$} & $6.761 \mathrm{e}-04$ \\
\hline Female Avatar: Male Participants v. Female & -5.09 & {$[-10.06,-0.08]$} & 0.019 \\
\hline \multicolumn{4}{|l|}{ Participants } \\
\hline Male Avatar: Male Participants v. Female & -5.04 & {$[-10.04,-0.11]$} & 0.013 \\
\hline \multicolumn{4}{|l|}{ Participants } \\
\hline Male Participants x Female Avatar v. Female & -5.45 & {$[-10.40,-0.43]$} & 0.015 \\
\hline \multicolumn{4}{|l|}{ Participants x Male Avatar } \\
\hline Female Participants,Female Avatar v. Male & 4.67 & {$[-0.19,9.72]$} & 0.01 \\
\hline Participants,Male Avatar & & & \\
\hline
\end{tabular}


Note. Results are described by means of the Median, the $95 \% \mathrm{HDI}$ and the $\mathrm{BF}_{10}\left(\mathrm{BF}_{10}>3\right.$ highlighted in bold).

\section{Figure 1}

Example of experimental stimuli

a)

Click the position on the line where you'd want the other person to stop

Covid-19 -

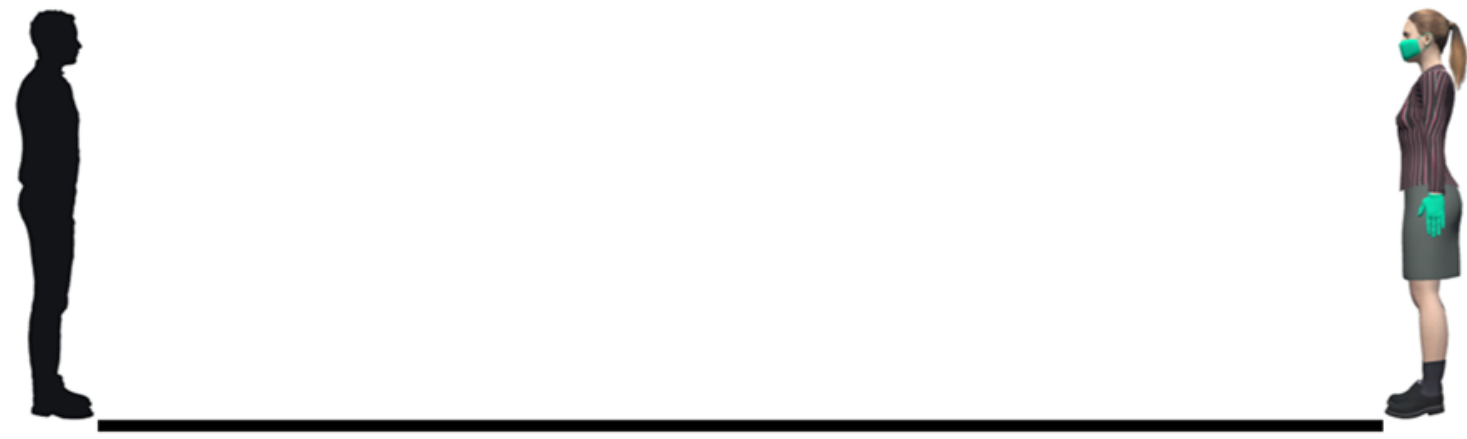

b)

Click the position on the line where you'd want the other person to stop

Covid-19+

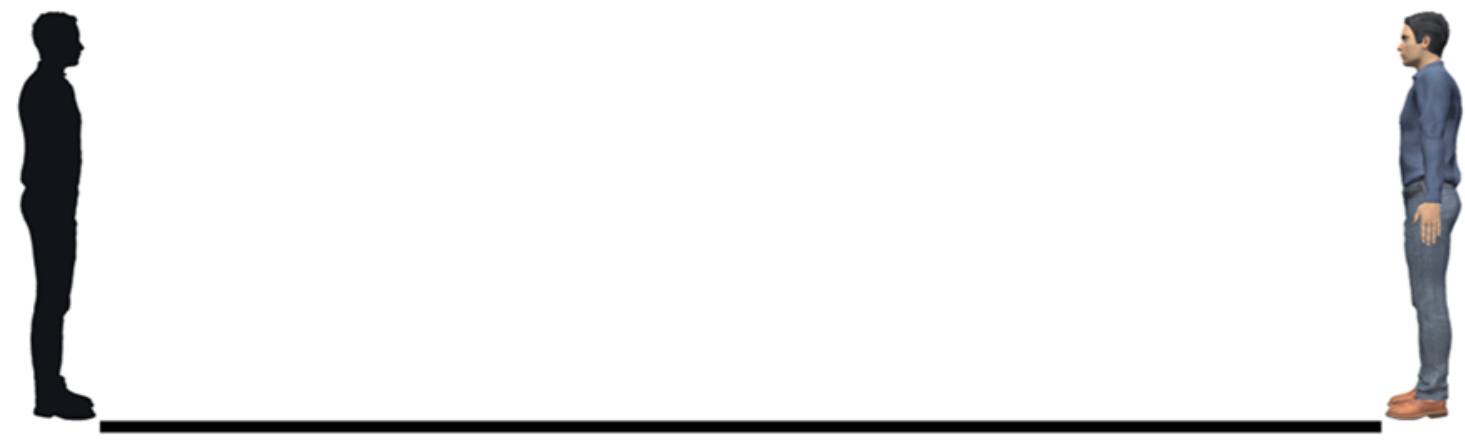

Note. Participants were instructed to imagine to be the person on the left side, represented by a gender-matched, black silhouette, and to indicate the distance to the other person (female or 
male, represented in panel $\mathbf{a}$ and $\mathbf{b}$, respectively) that they would feel comfortable keeping. The other person could be wearing protective equipment (a) or not (b). The label appearing on the upper part of the screen indicated the COVID-19 test result of the other person: a Negative test result was represented by a “_“" and shown in green (b); a Positive test result was represented by a "+" and displayed in red (a); an Unknown test result was represented by a "?" and displayed in gray (not shown).

Figure 2. Probability of direction and the magnitude of the effect for each predictor included in the study (y-axis) 


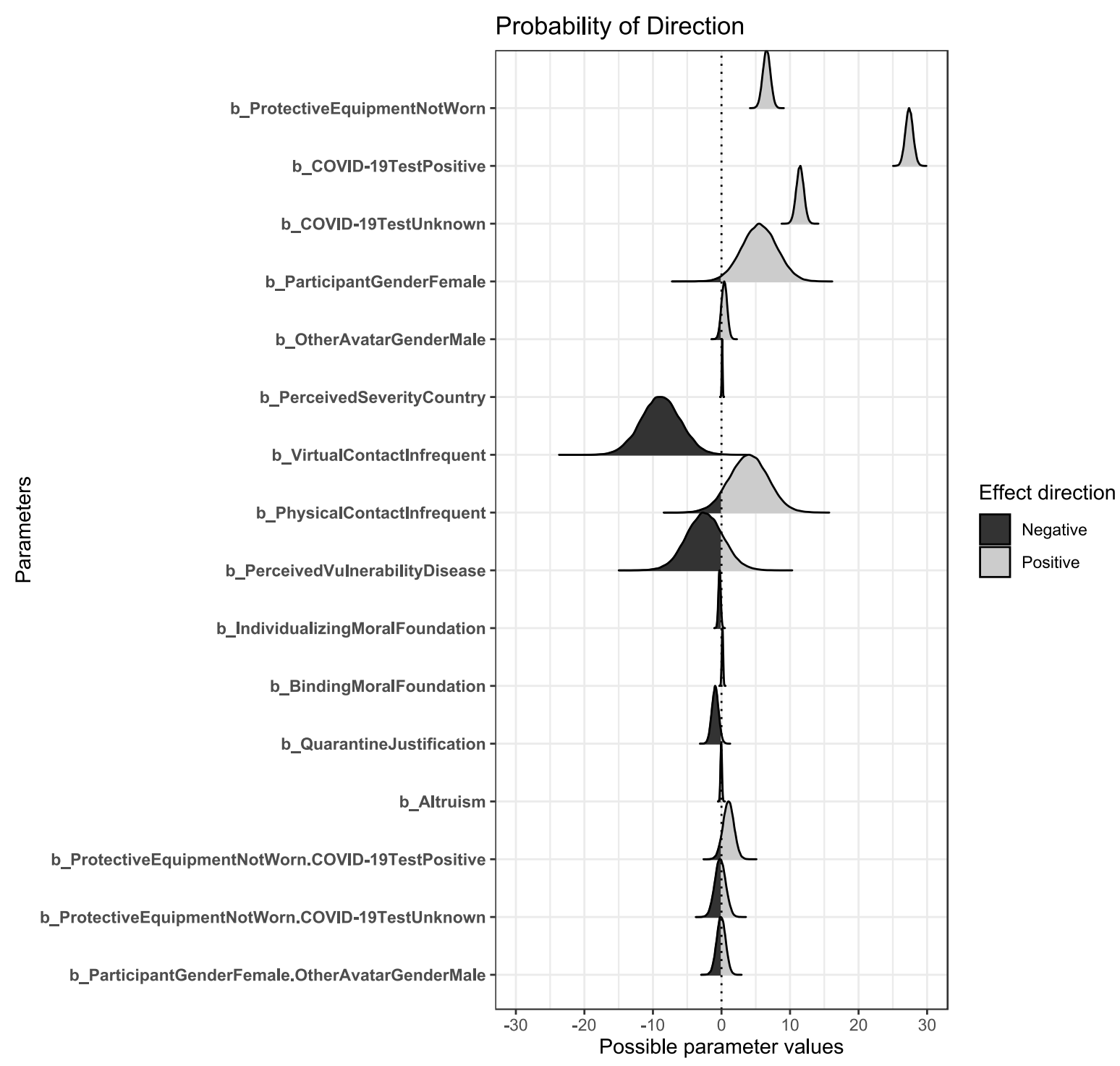

Note. The $\mathrm{x}$-axis indicates the possible parameter values. The color indicates the direction of the effect: black indicates a negative direction, while gray indicates a positive direction. The interaction between COVID-19 Test Result and Protective Equipment and the interaction between Participant's Gender and Other Avatar's Gender are better explained by the contrasts between all levels of the factors (COVID-19 Test Result: Protective Equipment see Table 4 and Figure 3; Participant's Gender: Other Avatar's Gender see Table 5).

\section{Figure 3}




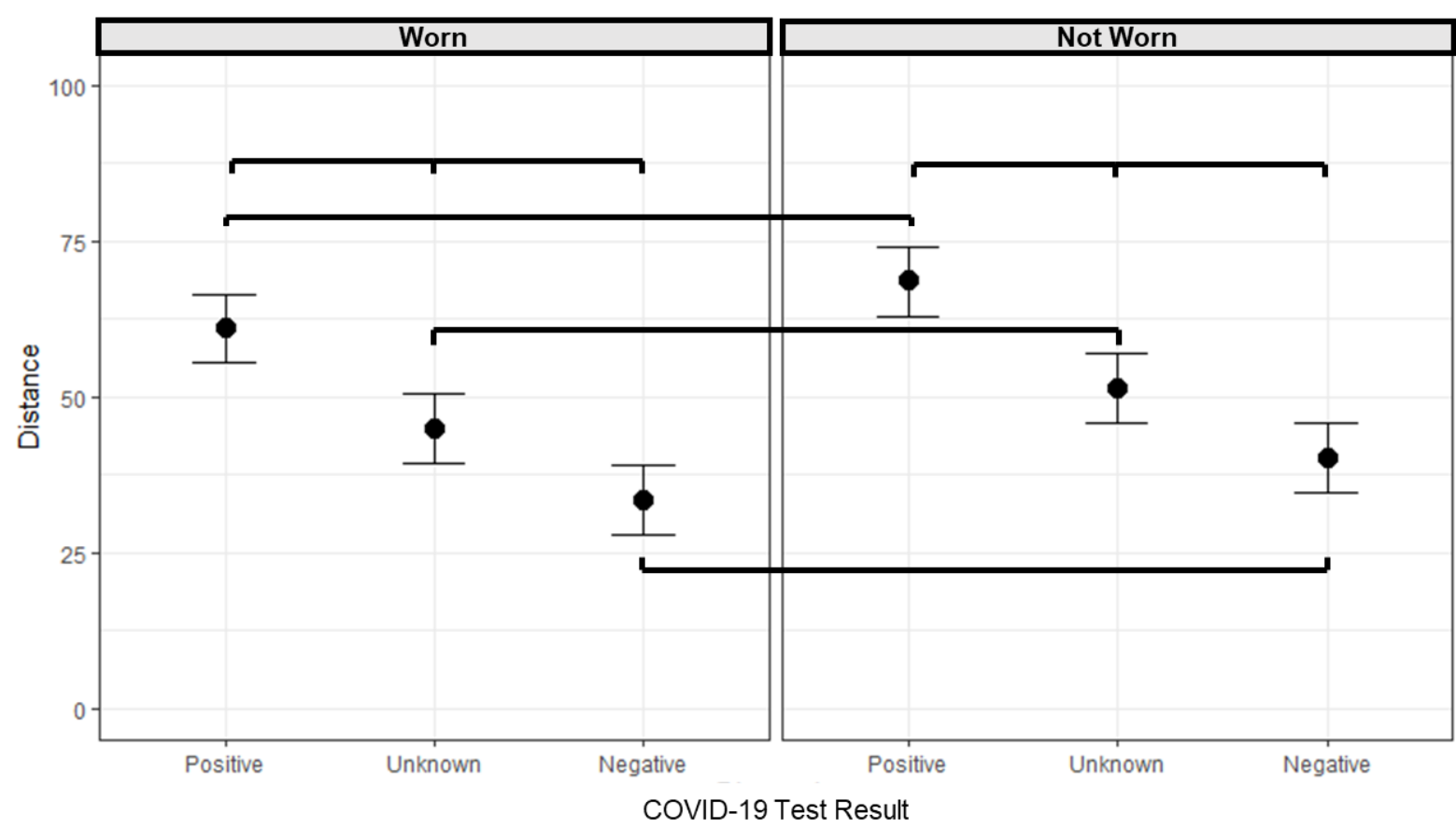

Note. The central dot indicates the posterior median and the whiskers indicate the lower and upper limits of the 95\% HDI. The contrasts for which the 95\% HDI does not include zero and for which $\mathrm{BF}_{10}>3$ (support for the alternative hypothesis) are connected with lines. 